\title{
DESEMPEÑO ECONÓMICO-FINANCIERO DE LOS DISTINTOS MODELOS EMPRESARIALES EN EL SECTOR DEL ACEITE DE OLIVA EN ESPAÑA
}

\section{ECONOMIC-FINANCIAL PERFORMANCE OF DIFFERENT BUSINESS MODELS IN THE SPANISH OLIVE OIL SECTOR}

\author{
Patricia Sepúlveda Orejuela (Universidad de Córdoba) ${ }^{1}$ \\ María Dolores Guerrero-Baena (Universidad de Córdoba) ${ }^{2}$ \\ José A. Gómez-Limón (Universidad de Córdoba) ${ }^{3}$
}

\begin{abstract}
Resumen:
Los objetivos principales de este trabajo son dos: en primer lugar, identificar los distintos modelos empresariales que existen en el sector del aceite de oliva en España y, en segundo lugar, realizar un diagnóstico del desempeño económico-financiero de cada uno de los modelos empresariales identificados. Los métodos empleados para conseguir los objetivos propuestos incluyen el análisis factorial, el análisis clúster y el análisis económico-financiero mediante el empleo de ratios. Los resultados del trabajo demuestran que en España existen cinco grandes patrones empresariales: i) grandes empresas orientadas a la exportación de aceite de oliva; ii) empresas de reducida dimensión exportadoras no sólo de aceite de oliva, sino también de otros productos complementarios; iii) almazaras de tipo mercantil que operan principalmente en el mercado nacional; iv) almazaras cooperativas orientadas al mercado nacional; y v) sociedades mercantiles cuya actividad principal es la distribución de aceite de oliva. De estos cincos patrones empresariales identificados, el más rentable y solvente corresponde al conformado por las sociedades mercantiles distribuidoras, mientras que el modelo de las grandes empresas exportadoras presenta graves problemas de viabilidad futura.
\end{abstract}

Palabras clave: Empresas de aceite de oliva; Análisis clúster; Análisis económico-financiero; Modelos empresariales; España.

Clasificación JEL: G39, L66.

\begin{abstract}
:
1 patricia.sepulveda@uco.es, Universidad de Córdoba.

2 dolores.guerrero@uco.es, Universidad de Córdoba.

3 jglimon@uco.es, Universidad de Córdoba.

Recibido 16 de noviembre de 2019. Aceptado 10 de marzo de 2020.
\end{abstract}

The main objectives of this paper are twofold: first, to identify the different business models that exist in the olive oil sector in Spain; and second, to provide an analysis of the economicfinancial performance of each identified business model. The methods used to achieve the two objectives include the factor analysis, the cluster analysis and the economic-financial analysis through the employment of ratios. The results of this paper show that in Spain there are five major business patterns or business models: i) large companies oriented to the olive oil exports; ii) small companies that export not only olive oil but also other complementary products; iii) commercial oil mills operating mainly in the national market; iv) cooperative oil mills oriented to the national market; and v) commercial companies whose main activity is the distribution of olive oil. Of these five business models identified, the most profitable and solvent corresponds 
to the one formed by the distribution companies, while the model of the large exporting companies presents serious problems of future viability.

Key words: Olive oil companies; Cluster analysis; Economic-financial analysis; Business models; Spain.

JEL Classification: G39, L66.

\section{INTRODUCCIÓN ${ }^{4}$}

España es el país líder mundial en producción y comercialización de aceite de oliva, concentrando la tercera parte de la superficie mundial de cultivo (2,4 millones de hectáreas) y más del 40 por ciento de la producción (entre 1,3 y 1,9 millones de toneladas, según años). Dentro del sector agrario español, la producción de aceite de oliva alcanza casi los 3.000 millones de euros anuales, cifra solo superada por la producción de los sectores de frutas y hortalizas (MAPA, 2019). España lidera igualmente los mercados internacionales de aceite de oliva, con exportaciones que oscilan entre 0,5 y 1,0 millones de toneladas, según las producciones anuales. Estos datos evidencian que se trata de un sector estratégico para el conjunto de la economía nacional y, de manera muy especial, para los territorios productores del sur peninsular.

La pujanza del sector del aceite de oliva en España está soportada por un entramado empresarial en el sector agroindustrial compuesto en la actualidad por 1.755 almazaras, 1.550 envasadoras y 22 refinerías de aceite, el 70 por ciento de las cuales cabe calificar como pequeñas o medianas empresas (pymes), cifra que demuestra la elevada fragmentación del sector. A pesar de este hándicap estructural, el conjunto del sector ha desarrollado una exitosa estrategia de internacionalización que permite la valorización de la creciente producción en los mercados exteriores. Entre los principales destinos del producto español se encuentran Italia, EEUU, Francia y Reino Unido (EXTENDA, 2017).

Mantener el liderazgo global del sector del aceite de oliva es una tarea compleja (Parras y Gómez-Limón, 2017), más aun teniendo en cuenta que, cada vez, son más los países que careciendo de tradición olivarera, pero siendo muy competitivos en costes, se incorporan a la actividad atraídos por la creciente demanda del producto (Vilar y Cárdenas, 2016). Este incremento de la oferta a nivel mundial conlleva, inevitablemente, tensiones en los mercados que se traducen en una bajada de los precios y de la rentabilidad empresarial de todos los miembros de una cadena de valor, ya de por sí inestable y con bajos márgenes comerciales (Briz et al., 2010; López-Pérez et al., 2018). A ello habría que sumarle la elevada incertidumbre a la que se enfrenta el sector en la actualidad motivada, principalmente, por las políticas arancelarias de uno de los principales países destino de las exportaciones, EE.UU.

Ante este panorama, conocer en profundidad y con precisión la estructura empresarial en la que se asienta el sector del aceite de oliva en España es fundamental para conseguir mantener e incrementar, mediante las medidas adecuadas, la competitividad y la rentabilidad del sector en su conjunto. Por ello, los principales objetivos de este trabajo son: por un lado, identificar los distintos patrones o modelos empresariales que coexisten en el sector del aceite de oliva en España a través de la caracterización de una muestra de empresas en función de un amplio y variado conjunto de criterios, empleando para ello el análisis factorial y el análisis clúster o de

\footnotetext{
${ }^{4}$ Los autores agradecen las sugerencias y comentarios realizados por los dos revisores anónimos, que han contribuido a la mejora del documento durante el proceso de revisión científica del mismo.
} 
conglomerados. Por otro lado, una vez identificados los distintos modelos empresariales, se aplica el análisis económico-financiero basado en ratios contables a cada conglomerado, para así poder diagnosticar el desempeño económico-financiero de cada uno de los modelos empresariales identificados.

A pesar de la relevancia del tema de investigación, debe indicarse que este trabajo es novedoso, en la medida que en la literatura no se han encontrado referencias con los mismos objetivos. En cualquier caso, como antecedentes cabe señalar, en primer lugar, trabajos que realizan análisis económico-financieros de empresas del sector del aceite de oliva a escala local para alguna tipología empresarial concreta, como son las cooperativas oleícolas (p. ej., Montegut et al., 2002). Y, en segundo lugar, también deben reseñarse algunos trabajos anteriores que se centran en analizar la relación entre varias variables relacionadas con el desempeño organizacional, como son los casos de García-Brenes (2005), donde se analiza la rentabilidad de las empresas industriales del sector del aceite de oliva, o de López-Pérez et al. (2018), donde se estudia la relación entre el fondo de maniobra y la rentabilidad de las empresas del sector oleícola en España.

Los resultados y conclusiones de este trabajo pueden ser de interés para un variado abanico de agentes relacionados con el sector del aceite de oliva. En primer lugar, para las empresas del propio sector, dado que aquí se presenta un análisis sobre el desempeño económico-financiero de cada tipo de empresas que operan dentro del mismo, por lo que los resultados aquí ofrecidos pueden servirles para realizar análisis comparativos con la competencia (benchmarking) respecto a aspectos claves como el grado de endeudamiento o la rentabilidad. En segundo lugar, tanto para inversores como para instituciones financieras, en la medida que en este trabajo pueden encontrar un apoyo documental para sus procesos de toma de decisiones relativas a la concesión de financiación a proyectos de inversión en el sector. En tercer lugar, la investigación puede resultar igualmente útil para los actuales y potenciales proveedores, suministradores de servicios y clientes (tanto nacionales como internacionales), quienes necesitan información actualizada sobre el desempeño económico-financiero del sector en su conjunto que dé soporte a sus operaciones comerciales. Y, en cuarto lugar, para las administraciones públicas, ya que esta investigación puede apoyar el diseño e implementación de políticas que tengan por objetivo incrementar la competitividad del sector del aceite de oliva y reforzar su estructura empresarial.

El presente trabajo se estructura en cinco apartados. Tras la Introducción, en el apartado 2 , se realiza una revisión de la literatura de los principales criterios para clasificar a las empresas. En el apartado 3, se detallan la muestra de empresas empleada y los métodos de análisis utilizados. En el apartado 4, se procede a la presentación y discusión de los resultados obtenidos. Y, por último, en el quinto apartado se presentan las conclusiones finales.

\section{CRITERIOS DE CLASIFICACIÓN DE EMPRESAS EN EL SECTOR DEL ACEITE OLIVA}

Para poder identificar los distintos modelos empresariales que coexisten en el sector del aceite de oliva español es necesario, en primer lugar, seleccionar una serie de criterios que permitan caracterizar a las empresas que operan dentro de este. Tras una extensa revisión de la literatura, se han recopilado los siguientes criterios de clasificación de empresas que se emplearán en el presente trabajo: tipo de actividad, dimensión, forma jurídica, pertenencia a grupo empresarial, mercado de actuación y realización de actividades complementarias. Todos estos criterios son de carácter general y ha sido ampliamente utilizados en la literatura empresarial, tanto para la tipificación de empresas agroalimentarias (p. ej., Domenech et al., 2014; Maté-Sánchez-Val y Harris, 2018), como en otros sectores de actividad (p. ej., Esteve- 
Pérez et al., 2010; Galetto et al., 2017). En cualquier caso, como se explica a continuación, estos criterios han sido adaptados al caso del sector del aceite de oliva, al objeto de establecer una tipología de empresas del sector adecuada para los objetivos de esta investigación.

\subsection{Tipo de actividad}

Uno de los criterios más relevantes para clasificar a las empresas que operan dentro del sector del aceite de oliva es según la actividad que desempeñan dentro de la cadena de valor (García-Brenes, 2005; Langreo, 2010; MAGRAMA, 2012). De manera resumida, las tres etapas que conforman la cadena de valor del aceite de oliva son: producción, industrialización y distribución del producto.

En primer lugar, en la actividad de producción, se lleva a cabo el cultivo y la recolección de la aceituna por parte de los agricultores, para su posterior envío a la molturación de la misma en las almazaras.

En segundo lugar, la fase de industrialización o transformación del producto puede, a su vez, abarcar varias subetapas. La primera es la fabricación o extracción de aceite, esto es, la transformación de la aceituna en aceite en almazaras, que pueden ser empresas privadas o de tipo mercantil, esto es, muelen la aceituna de agricultores ajenos a la empresa, o empresas cooperativas, que molturan la aceituna de sus socios. La segunda subetapa es la refinación de aceite, en la que, a partir del aceite lampante y mediante una serie de procesos físico-químicos, se obtiene el aceite de oliva refinado en empresas refinadoras. La tercera y última subetapa es el envasado del aceite, que se puede realizar en empresas envasadoras independientes (sólo dedicadas a esta última subetapa) o bien en envasadoras integradas en una almazara o en una refinería.

La tercera y última etapa de la cadena de valor del aceite de oliva es la distribución del producto, mediante la cual los productos elaborados se colocan en los mercados, bien siguiendo una estrategia de comercialización de "radio corto", esto es, vendiendo los productos en mercados locales, o bien siguiendo una estrategia de comercialización de "radio largo", esto es, realizando la venta de los productos en mercados alejados del núcleo productor-industrial, principalmente a través de hipermercados y supermercados o mediante el canal Horeca (hostelería, restauración y catering).

\subsection{Dimensión de la empresa}

En función de la dimensión o tamaño de la empresa, se puede diferenciar entre grandes empresas, pymes (pequeñas y medianas empresas) y microempresas. En este sentido, se ha considerado que la categoría de pymes está constituida, según lo dispuesto en el Reglamento (UE) $n^{0}$ 651/2014 de la Comisión, de 17 de junio de 2014, por las empresas que ocupan a menos de 250 personas y cuyo volumen de negocios anual no supera los 50 millones de euros o cuyo balance general anual no excede de 43 millones de euros. Por su parte, considerando lo establecido en la norma contable nacional (Real Decreto 1515/2007, de 16 de noviembre, por el que se aprueba el Plan General de Contabilidad de Pequeñas y Medianas Empresas y los criterios contables específicos para microempresas), se ha considerado como microempresas a aquellas que ocupan a menos de 10 personas y cuyo volumen de negocios anual no excede de 2 millones de euros o cuyo balance general anual no supera el millón de euros. Todas las empresas que no puedan caracterizarse como pymes o microempresas se considerarán grandes empresas. 


\subsection{Forma jurídica}

La elección de la forma jurídica de la empresa es una decisión relevante a considerar antes del inicio de la actividad, por su implicación sobre los objetivos de la organización y las estrategias a diseñar posteriormente. Se distinguen hasta veinte formas jurídicas ${ }^{5}$, que pueden ser agrupadas en las categorías de empresario individual y sociedad:

- Empresario individual: forma caracterizada, principalmente, por la no existencia de un mínimo legal de capital para la constitución de la empresa y por la asunción de responsabilidad del empresario ante los riesgos del negocio con todo su patrimonio personal. Desde la aprobación de la Ley 14/2013, de 27 de septiembre, de apoyo a los emprendedores y su internacionalización, se crea una nueva figura adicional, denominada "Emprendedor de responsabilidad limitada", en la que el empresario puede limitar de forma parcial la responsabilidad de sus deudas empresariales, descartando ciertos bienes personales como, por ejemplo, la vivienda habitual.

- Sociedad: entre las formas jurídicas asociativas se pueden encontrar sociedades civiles, sociedades mercantiles y otras formas asociativas.

- Sociedades civiles: se caracterizan por la necesidad de ser constituidas con un mínimo de dos socios (en algunos casos tres), que responden de manera ilimitada ante los riesgos de la sociedad, aunque no es necesario que exista un mínimo legal de capital para ser constituidas. La sociedad agraria de transformación (SAT) es un tipo de sociedad civil muy común en el sector agrario.

- Sociedades mercantiles que pueden, a su vez, agruparse en sociedades mercantiles personalistas y sociedades mercantiles capitalistas: En las primeras, los socios responden de manera ilimitada y personal de las deudas de la sociedad (es el caso de la sociedad colectiva y la sociedad comanditaria). En las segundas, los socios únicamente responden de las deudas sociales hasta el límite de su aportación al capital social, distinguiéndose las sociedades de responsabilidad limitada, las limitadas de formación sucesiva, las sociedades de responsabilidad limitada nueva empresa, las sociedades laborales, las sociedades anónimas y las sociedades comanditarias por acciones.

- Otras formas asociativas, tales como las comunidades de bienes, las agrupaciones de interés económico o las sociedades de economía social, como las cooperativas.

\subsection{Pertenencia a grupo empresarial}

Tomando como referencia la Directiva 2013/34/UE del Parlamento Europeo y del Consejo de 26 de junio de 2016, se pueden establecer, según su artículo 2, las siguientes definiciones de empresas en función de si pertenecen o no a un grupo empresarial. Por un lado, entre las empresas que forman parte de un grupo empresarial, se puede distinguir entre la sociedad matriz o sociedad dominante y las sociedades filiales, dominadas o dependientes. La primera es una sociedad de tipo mercantil que ejerce un control sobre una o varias empresas filiales, bien porque posea la mayoría de los derechos de voto de los accionistas o socios de la filial, o bien porque tenga el derecho de designar o desvincular de su cargo a la mayor parte de

\footnotetext{
${ }^{5}$ Ministerio de Industria, Comercio y Turismo, Secretaría General de Industria y de la Pequeña y Mediana Empresa: http://www.ipyme.org/esES/DecisionEmprender/FormasJuridicas/Paginas/FormasJuridicas.aspx
} 
los miembros del órgano de administración de la filial. Por otro lado, las empresas no pertenecientes a ningún grupo empresarial son independientes y ejercen su actividad y gestión del negocio de manera autónoma.

\subsection{Mercado de actuación}

Teniendo en cuenta el mercado en el que opera una empresa, esta puede hacerlo en el mercado nacional o, bien, comercializar su producto en los mercados internacionales como empresa exportadora y/o importadora. Así, aunque jurídicamente las operaciones comerciales con otros estados miembros pertenecientes a la UE son "operaciones intracomunitarias" y no exportaciones/importaciones (términos reservados a las operaciones con países terceros), en el presente trabajo se considera que una empresa "exportadora" es aquella que comercializa sus productos en el mercado europeo y/o en otros mercados internacionales. Este criterio es ampliamente utilizado en trabajos empíricos que analizan la internacionalización de las empresas, habiendo sido utilizado igualmente en el caso del sector del aceite de oliva en España (Esteban de la Rosa, 2015).

\subsection{Realización de actividades complementarias}

Para evitar que el aceite de oliva sea considerado un producto commodity, esto es, de escasa diferenciación y bajo valor añadido, muchas empresas están apostando por añadir valor a la producción, llevando a cabo estrategias de diversificación, tanto de carácter concéntrico o relacionadas con la producción principal, como no relacionadas con la producción de aceite de oliva. Las primeras son aquellas que tratan de aumentar el valor de la producción a través de la oferta de nuevos productos que, en cierto modo, guardan una estrecha relación con el inicialmente existente, logrando una sinergia entre las distintas actividades del negocio. Algunos ejemplos de este tipo de estrategias podrían ser la obtención de compuestos de los subproductos del olivar para su utilización en la industria agroalimentaria (conservas), así como para productos cosméticos, el uso energético de los subproductos del olivar (hueso o pellet, entre otros) o la realización de actividades turísticas (Rodríguez-Cohard y Parras, 2011; Gallardo-Cobos y Sánchez-Zamora, 2017). Las segundas, las estrategias de diversificación no relacionadas, se basan en ofrecer un nuevo producto o servicio no relacionado con la actividad oleícola (Gómez-Limón y Parras, 2017). Dada la relevancia de estas estrategias en el desempeño económico-financiero de las empresas, se ha considerado oportuno considerar si la empresa realiza o no actividades complementarias a la actividad principal como otro criterio de clasificación relevante.

Además de los seis criterios de catalogación anteriormente expuestos, se considerará también la localización de la empresa según la comunidad autónoma de pertenencia, al objeto de incluir la componente territorial en el análisis a realizar. Este criterio de clasificación ha sido adoptado frecuentemente en la literatura que analiza el sector del aceite de oliva en España (p. ej., Parras y Torres, 1996).

\section{FUENTE DE DATOS Y MÉTODOS EMPLEADOS EN EL ANÁLISIS}

\subsection{Muestra de empresas}

Para seleccionar la muestra de empresas objeto de estudio en este trabajo se ha empleado la base de datos SABI (Sistema de Análisis de Balances Ibéricos), de la empresa Bureau Van Dijk (https://sabi.bvdinfo.com). Se trata de una base de datos ampliamente utilizada en estudios centrados en la industria agroalimentaria (p. ej., Zouaghi et al., 2017; Martínez-Victoria et al., 
2019), que recopila las cuentas anuales, así como otra información económica, de casi dos millones de empresas españolas y portuguesas. La idoneidad de esta fuente para acometer los objetivos planteados en este trabajo radica principalmente en la gran cantidad de información disponible a nivel de empresa individual, así como en la estandarización de esta.

En un primer momento, para formar la muestra de empresas, se consideraron todas las empresas cuya actividad primaria o secundaria se correspondía con la clase 1043 (Fabricación de aceites de oliva) de la Clasificación Nacional de Actividades Económicas (CNAE-2009). Aunque un total de 910 empresas aparecían en SABI que cumplían este criterio, fue necesario excluir de este grupo a un total de 356 empresas que se encontraban en un estado de inactividad o extinción. Por ello, el tamaño final de la muestra para realizar el estudio ascendió a 554 empresas. Dado el elevado tamaño muestral así generado, se puede afirmar que esta muestra supone una buena representación del conjunto de la población analizada.

Una vez determinada la muestra de empresas del sector, se procedió a descargar toda la información disponible de la mencionada base de datos, tanto de carácter jurídico (forma jurídica, domicilio social, etc.), como de carácter económico-financiero (cuentas anuales de los ejercicios comprendidos entre 2015 y 2017). Esta información constituye la base de análisis cuantitativo realizado en este trabajo.

\subsection{Identificación de modelos empresariales}

El método seguido en este trabajo para alcanzar el objetivo propuesto en relación con la tipología de empresas del sector puede dividirse en varias etapas. En primer lugar, cada una de las 554 empresas de la muestra ha sido codificada en una base de datos en función de cada uno de los criterios de clasificación (tipo de actividad, dimensión, forma jurídica, pertenencia a grupo empresarial, mercado de actuación, realización de actividades complementarias y localización), al objeto de poder contar con la caracterización completa de las empresas del sector del aceite de oliva en España.

En segundo lugar, se ha utilizado el análisis factorial para reducir la dimensión de las variables recogidas en la base de datos construida (criterios de caracterización previamente expuestos) y, así, obtener los factores que agrupen a las variables que presenten mayor correlación entre sí. Más concretamente, el método empleado en el análisis factorial ha sido el análisis de componentes principales (ACP). Para medir la adecuación de la muestra, es decir, para saber si se pueden factorizar las variables originales de una manera eficiente, se han utilizado los estadísticos KMO (Kaiser-Meyer-Olkin) y la prueba de esfericidad de Bartlett (Hair et al., 2014).

En tercer lugar, se ha llevado a cabo un análisis clúster, técnica multivariante cuya intención es agrupar casos en función de las características que estos presentan. Así, en este trabajo, se pretenden obtener grupos heterogéneos entre sí que incluyan empresas que presenten características similares. De este modo, empleando como variables clasificadoras los factores obtenidos del análisis factorial, se ha asignado cada empresa a un clúster o conglomerado concreto.

Para la obtención de los conglomerados (tipologías de empresas) se ha utilizado un procedimiento jerárquico, considerando la distancia Euclídea al cuadrado como indicador para medir la similitud entre los distintos casos (empresas) (Kettenring, 2006). Para aplicar esta técnica clúster se han tomado como criterios tipificadores los factores que resultan más relevantes de los obtenidos a través del análisis factorial. Asimismo, para la agrupación de casos se ha aplicado el método de Ward, el cual agrupa sucesivamente casos y grupos de manera que se minimice el incremento de varianza residual global, tendiendo a formar clústeres esféricos y de tamaño similar (Hair et al., 2014). 
Para establecer las características definitorias de cada uno de los conglomerados obtenidos mediante el método antes descrito, se han comparado los valores medios (variables métricas) o las frecuencias relativas (variables categóricas) de los distintos clústeres respecto a las diferentes variables tipificadoras, empleando para ello el test de comparación de medias ANOVA y el test de comparación de frecuencias Chi-Cuadrado, respectivamente. El contraste de ambas pruebas está conformado por la hipótesis nula de que las medias o frecuencias relativas de los $k$ conglomerados son iguales, frente a la hipótesis alternativa de que al menos uno de los clústeres es distinto a los demás.

Los conglomerados resultantes del análisis clúster constituirán las unidades del posterior análisis económico-financiero. Dado que la información que proporciona la base de datos SABI es de carácter individual a nivel de empresa, se ha hecho uso de la herramienta "Agregación" disponible en la propia base de datos al objeto de poder generar los estados contables agregados de cada clúster. Tales cuentas anuales agregadas han sido analizadas como si se tratase de una empresa "ficticia” representativa o “tipo" de cada clúster, permitiendo el diagnóstico del desempeño económico-financiero de cada modelo empresarial.

\subsection{Análisis económico-financiero}

Para poder realizar el diagnóstico económico-financiero de las empresas tipo de cada clúster a partir de sus cuentas anuales se ha aplicado el análisis de ratios. En concreto, se han calculado un total de 32 ratios contables, tal y como se puede observar en la tabla 1, que proporcionan información pormenorizada sobre los distintos aspectos de la situación económico-financiera de las empresas: liquidez, endeudamiento, rotaciones de elementos del activo, análisis de la cuenta de pérdidas y ganancias, rentabilidad y fondo de maniobra.

TABLA 1. RATIOS ECONÓMICO-FINANCIERAS Y VALORES IDEALES

\begin{tabular}{|l|c|}
\hline \multicolumn{1}{|c|}{ RATIOS } & $\begin{array}{c}\text { VALORES } \\
\text { IDEALES }\end{array}$ \\
\hline LIQUIDEZ & $1,5-2,0$ \\
\hline Liquidez: Activo corriente / Pasivo corriente & $\sim 1$ \\
\hline Tesorería: (Realizable + Tesorería) / Pasivo corriente & $0,2-0,3$ \\
\hline Prueba ácida: Disponible / Pasivo corriente & Positivo \\
\hline Fondo de maniobra (€): Activo corriente - Pasivo corriente & $0,4-0,6$ \\
\hline ENDEUDAMIENTO & $0,7-1,5$ \\
\hline $\begin{array}{l}\text { Endeudamiento: (Pasivo no corriente + Pasivo corriente) / (Patrimonio neto + Pasivo } \\
\text { no corriente + Pasivo corriente) }\end{array}$ & $1,5-2,5$ \\
\hline Autonomía financiera: Fondos propios / (Pasivo no corriente + Pasivo corriente) & Reducido \\
\hline Solvencia: Activo / (Pasivo no corriente + Pasivo corriente) & $\leq i_{\text {mercado }}$ \\
\hline Calidad de la deuda: Pasivo corriente / (Pasivo no corriente + Pasivo corriente) & Reducido \\
\hline Coste de la deuda (\%): Gastos financieros / Deuda con coste & \\
\hline $\begin{array}{l}\text { Coste medio del pasivo (\%): (Gastos financieros + Dividendos) / (Patrimonio Neto + } \\
\text { Pasivo no corriente + Pasivo corriente) }\end{array}$ & Elevado \\
\hline ROTACIONES & Elevado \\
\hline Rotación del Activo no corriente (ANC): Ventas / Activo no corriente & Elevado \\
\hline Rotación del Activo corriente (AC): Ventas / Activo corriente & Elevado \\
\hline Rotación del Activo: Ventas / Activo & Elevado \\
\hline Rotación de Existencias: Ventas / Existencias & \\
\hline Rotación de Clientes: Ventas / Clientes & \\
\hline
\end{tabular}




\begin{tabular}{|c|c|}
\hline \multicolumn{2}{|l|}{ CUENTA DE PÉRDIDAS Y GANANCIAS } \\
\hline Crecimiento de Ventas (\%): Ventas $_{n} /$ Ventas $_{n-1}$ & Elevado \\
\hline 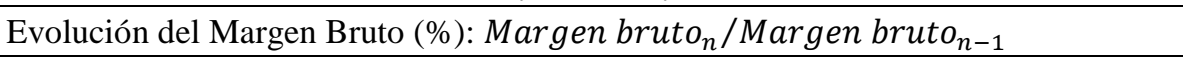 & Elevado \\
\hline Evolución del Beneficio antes de intereses e impuestos (BAII) (\%): $B A I I_{n} / B A I I_{n-1}$ & Elevado \\
\hline Evolución del Beneficio antes de impuestos (BAI) (\%): $B A I_{n} / B A I_{n-1}$ & Elevado \\
\hline Evolución del Beneficio neto (BN) (\%): $B N_{n} / B N_{n-1}$ & Elevado \\
\hline Punto de equilibrio (€): Gastos fijos / (1 - Gastos variables sobre ventas) & Elevado \\
\hline $\begin{array}{l}\text { Cobertura umbral de rentabilidad (UR): Importe neto cifra de negocios / Punto } \\
\text { equilibrio }\end{array}$ & $>1$ \\
\hline Apalancamiento operativo: Variación BAII (\%) / Variación ventas (\%) & Elevado \\
\hline \multicolumn{2}{|l|}{ RENTABILIDAD } \\
\hline Rentabilidad del Activo (\%): BAII / Activo total & Elevado \\
\hline Rentabilidad de las Ventas (\%): Resultado del ejercicio / Ventas & Elevado \\
\hline Rentabilidad Financiera (\%): Beneficio Neto / Fondos propios & Elevado \\
\hline Margen de Ventas (\%): BAII / Ventas & Elevado \\
\hline Apalancamiento Financiero: (Activo / Fondos propios) $\times(B A I / B A I I)$ & Elevado \\
\hline Autofinanciación: Resultado del ejercicio + Amortizaciones - Dividendos & Elevado \\
\hline Autofinanciación sobre Ventas (\%): Autofinanciación / Ventas & Elevado \\
\hline \multicolumn{2}{|l|}{ FONDO DE MANIOBRA } \\
\hline $\begin{array}{l}\text { Necesidades operativas de fondos (NOF) (€): Activo corriente operativo - Pasivo } \\
\text { corriente operativo }\end{array}$ & $<\mathrm{FM}$ \\
\hline Cobertura de las NOF: Fondo de maniobra / NOF & $\geq 1$ \\
\hline Fuente: Amat (2008). & \\
\hline
\end{tabular}

\section{RESULTADOS}

\subsection{Descripción de la muestra de empresas del sector}

La tabla 2 muestra los datos descriptivos de la muestra de empresas del sector considerada en este trabajo. Atendiendo al criterio del tipo de actividad, se puede observar que, de las 554 empresas que componen la muestra, el 76,5 por ciento se dedican a las tres fases de la cadena de valor, mientras que el 20,8 por ciento se dedica a la industrialización y a la distribución. El grueso del sector lo componen microempresas (58,1 por ciento) y pymes (39,0 por ciento), siendo en su mayoría sociedades mercantiles (86,1 por ciento), mientras que las sociedades cooperativas suponen el 12,6 por ciento ${ }^{6}$.

Aunque las empresas independientes son el 67,3 por ciento de la muestra, no es desdeñable el número de empresas que pertenecen a un grupo empresarial, como matrices o filiales (32,7 por ciento). Según el mercado de actuación, más de la mitad de las empresas opera únicamente en el mercado nacional, siendo mayoritarias las empresas que realizan algún tipo de actividad complementaria a la actividad principal (57,6 por ciento). Finalmente, en cuanto a su distribución territorial, destaca la Comunidad Autónoma de Andalucía, donde tienen su domicilio fiscal el 48,6 por ciento de las empresas de la muestra. Le siguen a distancia CastillaLa Mancha (11,9 por ciento) y Cataluña (10,3 por ciento).

\footnotetext{
${ }^{6}$ Como limitación del trabajo, cabe destacar la baja representación de las sociedades cooperativas en el conjunto de la muestra de empresas. Este hecho viene motivado porque la base de datos SABI se nutre de la información depositada (cuentas anuales) por las empresas en los registros mercantiles, depósito que resulta obligatorio para las sociedades mercantiles, pero no para las cooperativas.
} 


\begin{tabular}{|c|c|c|c|}
\hline \multicolumn{4}{|c|}{$\begin{array}{l}\text { TABLA 2. DESCRIPCIÓN DE LA MUESTRA SEGÚN CRITERIOS DE } \\
\text { CLASIFICACIÓN }\end{array}$} \\
\hline Criterio & Categoría & $\mathbf{N}^{\circ}$ empresas & \% muestra \\
\hline \multirow{7}{*}{ Tipo de actividad } & Sólo producción & 0 & $0,0 \%$ \\
\hline & Sólo industrialización & 0 & $0,0 \%$ \\
\hline & Sólo distribución & 15 & $2,7 \%$ \\
\hline & Producción e industrialización & 0 & $0,0 \%$ \\
\hline & Industrialización y distribución & 115 & $20,8 \%$ \\
\hline & Producción, industrialización y distribución & 424 & $76,5 \%$ \\
\hline & Total & 554 & $100,0 \%$ \\
\hline \multirow{4}{*}{$\begin{array}{l}\text { Dimensión de la } \\
\text { empresa }\end{array}$} & Grande & 16 & $2,9 \%$ \\
\hline & Pyme & 216 & $39,0 \%$ \\
\hline & Microempresa & 322 & $58,1 \%$ \\
\hline & Total & 554 & $100,0 \%$ \\
\hline \multirow{5}{*}{ Forma jurídica } & Sociedad civil & 0 & $0,0 \%$ \\
\hline & Sociedad mercantil (SA y SL) & 477 & $86,1 \%$ \\
\hline & Sociedad cooperativa & 70 & $12,6 \%$ \\
\hline & Sociedad agraria de transformación & 7 & $1,3 \%$ \\
\hline & Total & 554 & $100,0 \%$ \\
\hline \multirow{4}{*}{$\begin{array}{l}\text { Pertenencia a un } \\
\text { grupo empresarial }\end{array}$} & Sí pertenece como matriz & 109 & $19,7 \%$ \\
\hline & Sí pertenece como filial & 72 & $13,0 \%$ \\
\hline & No pertenece: independiente & 373 & $67,3 \%$ \\
\hline & Total & 554 & $100,0 \%$ \\
\hline \multirow{5}{*}{ Mercado de actuación } & Sólo mercado nacional & 334 & $60,3 \%$ \\
\hline & Importadora & 5 & $0,9 \%$ \\
\hline & Exportadora & 161 & $29,1 \%$ \\
\hline & Exportadora e importadora & 54 & $9,7 \%$ \\
\hline & Total & 554 & $100,0 \%$ \\
\hline \multirow{3}{*}{$\begin{array}{l}\text { Realización de } \\
\text { actividades } \\
\text { complementarias }\end{array}$} & No realiza actividades complementarias & 235 & $42,4 \%$ \\
\hline & Sí realiza actividades complementarias & 319 & $57,6 \%$ \\
\hline & Total & 554 & $100,0 \%$ \\
\hline \multirow{14}{*}{ Localización } & Andalucía & 269 & $48,6 \%$ \\
\hline & Aragón & 29 & $5,2 \%$ \\
\hline & Castilla-La Mancha & 66 & $11,9 \%$ \\
\hline & Castilla-León & 6 & $1,1 \%$ \\
\hline & Cataluña & 57 & $10,3 \%$ \\
\hline & Comunidad de Madrid & 32 & $5,8 \%$ \\
\hline & Comunidad Foral de Navarra & 8 & $1,4 \%$ \\
\hline & Comunidad Valenciana & 28 & $5,1 \%$ \\
\hline & Extremadura & 36 & $6,5 \%$ \\
\hline & Galicia & 2 & $0,4 \%$ \\
\hline & Islas Baleares & 6 & $1,1 \%$ \\
\hline & La Rioja & 5 & $0,9 \%$ \\
\hline & Región de Murcia & 10 & $1,8 \%$ \\
\hline & Total & 554 & $100,0 \%$ \\
\hline
\end{tabular}

\subsection{Resultados de la clasificación de empresas}

En primer lugar, se comentan los resultados del análisis factorial. Como se ha descrito en el apartado 3.2, para reducir la dimensión de las variables clasificadoras se ha utilizado esta técnica estadística multivariante. Previamente, se ha analizado si se cumplen los requisitos para poder llevar a cabo este análisis (valores elevados del estadístico KMO y variables altamente correlacionadas), llegando a la conclusión de que ambos requisitos se cumplen: el valor del estadístico KMO es de 0,7 y la prueba de esfericidad de Bartlett es significativa (p-valor= 
0,000), rechazándose la hipótesis nula de no correlación de las variables. Dado que se cumplen los requisitos, se ha podido realizar el análisis factorial. En la tabla 3 se pueden observar los resultados obtenidos al aplicar esta técnica. Las variables originales son agrupadas en once factores, de los cuales únicamente se tomarán en consideración los cinco primeros, correspondientes a aquellos que poseen un autovalor superior a 1 (Hair et al., 2014). Escoger los factores con autovalores elevados está relacionado directamente con la varianza pues, a mayor autovalor del factor, mayor será la varianza explicada por dicho factor. De esta manera, al retener para el posterior análisis los cinco primeros factores se considerará dos terceras partes (66,6 por ciento) de la varianza explicada por el conjunto de las variables tipificadoras.

\begin{tabular}{|c|ccc|}
\hline \multicolumn{4}{|c|}{ TABLA 3. RESULTADOS DEL ANÁLISIS FACTORIAL } \\
\cline { 2 - 5 } FACTOR & \multicolumn{3}{|c|}{ AUTOVALORES INICIALES } \\
\cline { 2 - 4 } & Total & Porcentaje de varianza & Porcentaje de varianza acumulado \\
\hline $\mathbf{1}$ & $\mathbf{2 , 6}$ & $\mathbf{2 3 , 8}$ & $\mathbf{2 3 , 8}$ \\
$\mathbf{2}$ & $\mathbf{1 , 5}$ & $\mathbf{1 3 , 4}$ & $\mathbf{3 7 , 2}$ \\
$\mathbf{3}$ & $\mathbf{1 , 2}$ & $\mathbf{1 0 , 8}$ & $\mathbf{4 8 , 0}$ \\
$\mathbf{4}$ & $\mathbf{1 , 1}$ & $\mathbf{9 , 5}$ & $\mathbf{5 7 , 5}$ \\
$\mathbf{5}$ & $\mathbf{1 , 0}$ & $\mathbf{9 , 1}$ & $\mathbf{6 6 , 6}$ \\
6 & 0,9 & 8,1 & 74,7 \\
7 & 0,8 & 6,9 & 81,6 \\
8 & 0,7 & 6,4 & 98,0 \\
9 & 0,7 & 6,0 & 94,0 \\
10 & 0,4 & 3,9 & 100,0 \\
11 & 0,2 & 2,1 & \\
\hline \multicolumn{5}{|l}{} \\
\hline
\end{tabular}

A continuación, en la tabla 4 se muestra la matriz de componentes rotados obtenida a través del método de Varimax. Este método posibilita ver qué variables consideradas originalmente para la tipificación de empresas presentan mayores cargas en cada uno de los factores retenidos; es decir, facilita la identificación de qué factor se asocia con cada variable o con cada conjunto de variables.

\section{TABLA 4. MATRIZ DE COMPONENTES ROTADOS}

\begin{tabular}{|l|ccccc|}
\hline \multirow{2}{*}{ VARIABLES } & \multicolumn{5}{c|}{ FACTOR } \\
\cline { 2 - 6 } & $\mathbf{1}$ & $\mathbf{2}$ & $\mathbf{3}$ & $\mathbf{4}$ & $\mathbf{5}$ \\
\hline Pertenencia a grupo (0=No; 1=Sí) & $-0,08$ & $\mathbf{0 , 7 3}$ & 0,17 & 0,07 & $-0,07$ \\
Forma jurídica (0=cooperativas y SAT; 1=mercantiles) & $-0,14$ & 0,28 & $-0,28$ & $\mathbf{- 0 , 6 7}$ & $-0,19$ \\
Mercado de actuación (0=mercado nacional; & 0,26 & $\mathbf{0 , 7 0}$ & 0,11 & 0,02 & $-0,08$ \\
1=mercado internacional) & $\mathbf{0 , 8 1}$ & 0,13 & $-0,02$ & 0,08 & 0,02 \\
Número de trabajadores (número UTA*) & $\mathbf{0 , 8 9}$ & 0,10 & 0,01 & $-0,01$ & 0,01 \\
Total activo (millones $€$ ) & $\mathbf{0 , 8 9}$ & 0,12 & $-0,04$ & $-0,02$ & 0,01 \\
Volumen de negocios (millones $€$ ) & 0,07 & 0,43 & $-0,05$ & $\mathbf{0 , 6 2}$ & 0,13 \\
Actividad complementaria (0=No; 1=Sí) & $-0,13$ & $-0,06$ & $-0,15$ & $\mathbf{0 , 6 0}$ & $-0,33$ \\
Localización (0=Andalucía; 1=Otras CC.AA.) & $-0,01$ & $-0,18$ & $\mathbf{0 , 7 4}$ & 0,13 & 0,02 \\
Dedicada a actividad producción (0=No; 1=Sí) & $-0,04$ & 0,10 & $\mathbf{0 , 8 0}$ & $-0,12$ & $-0,02$ \\
Dedicada a actividad industrialización (0=No; 1=Sí) & $-0,00$ & 0,01 & $-0,02$ & 0,02 & $\mathbf{0 , 9 3}$ \\
Dedicada a actividad distribución (0=No; 1=Sí) & & & &
\end{tabular}

* Unidades de trabajo agrícola.

Fuente: Elaboración propia. 
A continuación, se explica cada uno de los factores considerados para el análisis en base a las principales variables definitorias de cada uno de ellos:

- Factor 1 (tamaño). El primer factor, que hace referencia al tamaño o la dimensión empresarial, explica el 23,8 por ciento de la varianza. Las variables que principalmente afectan a este factor son las relacionadas con los aspectos que determinan el tamaño empresarial: número de trabajadores, cifra total de activo y volumen de negocios.

- Factor 2 (pertenencia a grupo y comercio exterior). Las variables que presentan mayores cargas en este factor son pertenencia a grupo empresarial y mercado de actuación que, en conjunto, explican el 13,4 por ciento de la varianza. Ambas variables presentan cargas elevadas y positivas: las empresas que pertenecen a un grupo empresarial tienen más posibilidades de realizar actividades de exportación, mientras que aquellas que son independientes con más probabilidad se dedicarán a la venta en territorio nacional.

- Factor 3 (empresas productoras e industriales). Este factor explica el 10,8 por ciento de la varianza total, estando definido principalmente por dos variables: dedicada a la actividad de producción y dedicada a la actividad de industrialización. Ambas variables presentan cargas elevadas positivas. Por tanto, se puede afirmar que las empresas dedicadas a la producción tienen alta probabilidad de realizar también la transformación del producto.

- Factor 4 (forma jurídica, actividad complementaria y localización). Este factor explica el 9,5 por ciento de la varianza total. Los dos factores con mayor carga positiva son actividad complementaria y localización, mientras que la forma jurídica también es otra variable relevante, pero presenta una carga negativa. Esto indica que, por lo general, las sociedades cooperativas y las sociedades agrarias de transformación (valor 0 de la variable "forma jurídica”) suelen realizar actividades complementarias (valor 1 de la variable "actividad complementaria”) y, con mayor probabilidad, están situadas fuera de Andalucía (valor 1 de la variable "localización”).

- Factor 5 (Empresas distribuidoras). El último factor a tener en cuenta en el estudio explica el 9,1 por ciento de la varianza y presenta como variable más relevante dedicada a la actividad de distribución.

Una vez determinados los factores que servirán de variables de clasificación, se ha procedido a aplicar el análisis clúster. A la vista del dendrograma que se obtiene, se ha considerado conveniente clasificar la muestra de empresas en cinco conglomerados o clústeres, que representan cada uno de los patrones o modelos empresariales. La tabla 5 muestra la importancia relativa de cada uno de los clústeres, según el número de empresas y la cifra de ventas que comprende cada uno. 


\begin{tabular}{|c|c|c|c|c|}
\hline \multicolumn{5}{|c|}{ TABLA 5. CLÚSTERES OBTENIDOS SEGÚN FACTORES } \\
\hline Clúster & Frecuencia & Porcentaje & $\begin{array}{c}\text { Cifra de ventas } \\
\text { (millones } €)\end{array}$ & $\begin{array}{c}\text { Porcentaje cifra ventas } \\
\text { sobre el sector }\end{array}$ \\
\hline 1 & 6 & $1,1 \%$ & 3.433 & $42,6 \%$ \\
\hline 2 & 219 & $39,5 \%$ & 3.481 & $43,2 \%$ \\
\hline 3 & 238 & $43,0 \%$ & 622 & $7,7 \%$ \\
\hline 4 & 74 & $13,3 \%$ & 125 & $1,6 \%$ \\
\hline 5 & 17 & $3,1 \%$ & 396 & $4,9 \%$ \\
\hline Total & 554 & $100,0 \%$ & 8.057 & $100,0 \%$ \\
\hline
\end{tabular}

Las pruebas ANOVA y Chi-Cuadrado han permitido rechazar la hipótesis nula de igualdad de medias/frecuencias, lo que ha posibilitado establecer las características diferenciales que definen a cada uno de los conglomerados o clústeres. Las conclusiones obtenidas de estas pruebas se detallan a continuación:

- Clúster 1 (Grupos empresariales exportadores). Este clúster es el menos numeroso de todos, ya que las empresas integradas en el mismo únicamente representan el 1,1 por ciento del total de empresas de la muestra, aunque es el conglomerado más importante atendiendo al porcentaje de cifra de ventas que acumula sobre el total (42,6 por ciento). Las variables que resultan más significativas en este conglomerado son el tamaño (grandes empresas), la clara vocación por el comercio internacional y la pertenencia a un grupo empresarial. Igualmente, también se puede afirmar que las empresas incluidas en este clúster se caracterizan por dedicarse a la realización de actividades complementarias a la fabricación de aceite de oliva.

- Clúster 2 (Pymes exportadoras). Este clúster agrupa al 39,5 por ciento de la muestra (43,2 por ciento de ventas sobre el total) y está formado por pequeñas y medianas empresas de tipo mercantil. Las empresas de este clúster se dedican a las tres actividades de la cadena de valor (producción, transformación y distribución del producto) y se caracterizan por su fuerte orientación al mercado exterior, exportando tanto aceite de oliva como otros productos complementarios.

- Clúster 3 (Pequeñas almazaras mercantiles orientadas al mercado nacional). Las empresas que conforman este clúster representan el 43,0 por ciento del total de la muestra, por lo que es el conglomerado más numeroso, pero sólo concentran el 7,7 por ciento de las ventas totales. Este clúster se caracteriza principalmente por reunir a pymes y microempresas con una forma jurídica de sociedad mercantil, dedicadas exclusivamente a la actividad oleícola, principalmente a la transformación de la aceituna en aceite de oliva y a la distribución del producto en mercados nacionales.

- Clúster 4 (Almazaras cooperativas orientadas al mercado nacional). Este conglomerado de empresas supone el 13,3 por ciento del total de empresas de la muestra y sólo el 1,6 por ciento de las ventas. Está formado por pequeñas cooperativas y sociedades agrarias de transformación, que desarrollan todas las fases de la cadena de valor y apenas están presentes en los mercados internacionales.

- Clúster 5 (Sociedades mercantiles distribuidoras). Este quinto clúster agrupa al 3,1 por ciento de las empresas de la muestra, que reúnen el 4,9 por ciento de las ventas totales. Se caracteriza por agrupar principalmente a sociedades mercantiles que se dedican sólo a la distribución del producto (última fase de la cadena de valor), ubicadas en su mayoría en Cataluña, comunidad en la que el cultivo del olivar es muy bajo, hecho que explica la menor actividad productora. 


\subsection{Resultados del análisis económico-financiero}

En este apartado, se muestran y discuten los resultados del análisis económico-financiero, tanto del sector del aceite de oliva en su conjunto (empresa "virtual” construida agregando las cuentas anuales de la totalidad de empresas de la muestra), como de cada uno de los clústeres creados, atendiendo a la situación de liquidez, endeudamiento, rotaciones, cuenta de resultados, rentabilidad y necesidades operativas de fondos (véase tabla 6).

\section{a) Análisis de la liquidez}

En el sector en su conjunto se observan valores de las ratios que miden la liquidez de la empresa en el periodo 2015-2017 algo por debajo de lo que resultaría ideal, aunque en línea con los valores correspondientes a la industria de la alimentación en su conjunto en España (Banco de España, 2019). Un valor bajo de esta ratio implica que las deudas a corto plazo no son cubiertas lo suficientemente por los elementos más líquidos del activo, lo que podría dificultar el cumplimiento de los compromisos de pago en el corto plazo. Esta falta de liquidez se ve reflejada en todos los clústeres, a excepción del grupo de sociedades mercantiles distribuidoras (clúster 5) que, incluso presentó un valor de esta ratio por encima del valor óptimo en el ejercicio 2015, aunque corregido en ejercicios sucesivos. Un valor muy elevado, esto es, un exceso de liquidez, podría indicar la existencia de activos corrientes ociosos y, por tanto, activos con un coste de oportunidad considerable. Afortunadamente, la buena gestión de este conglomerado de empresas en los ejercicios posteriores a 2015 logró situar la ratio de liquidez en el intervalo ideal.

Analizando con detalle cada uno de los restantes clústeres, se observa que el grupo de las almazaras cooperativas orientadas al mercado nacional (clúster 4) presenta los peores valores de la ratio de liquidez en los ejercicios 2015 y 2016. En este sentido, se debe recordar que este conglomerado está formado por pequeñas cooperativas y sociedades agrarias de transformación cuya producción se destina principalmente al mercado nacional. Es un hecho que, en el mercado interior la presión de la marca de distribución está disminuyendo los precios del aceite de oliva, incluso por debajo del coste, a la vez que está alargando los plazos medios de pago a las almazaras. Ambos motivos justifican que sean las cooperativas y SATs orientadas principalmente al mercado nacional, empresas con bajo poder de negociación, las que mayores problemas de liquidez presenten (Ruiz et al., 2013).

\section{b) Análisis del endeudamiento}

En cuanto a las ratios relacionadas con el endeudamiento, el sector presenta un nivel de endeudamiento $(0,7)$ ligeramente por encima del endeudamiento de la industria de la alimentación en su conjunto (0,6) (Banco de España, 2019) y del valor ideal. Este resultado está en línea con el trabajo de López-Pérez et al. (2018) para el periodo 2010-2014, en el que se reportan valores igualmente cercanos a 0,7 para el sector del aceite de oliva. El primer y el cuarto clúster (grupos empresariales exportadores y almazaras cooperativas orientadas al mercado nacional) destacan por presentar las peores cifras de endeudamiento en comparación con el sector, lo que apunta a una posible descapitalización de estos dos grupos de empresas. Aunque, se ha de destacar que las almazaras cooperativas orientadas al mercado nacional mejoraron en 2017 su ratio de endeudamiento, situándolo en el límite superior del intervalo de valores ideales. Por su parte, el quinto clúster (sociedades mercantiles distribuidoras) presenta la situación más favorable de endeudamiento, con un adecuado equilibrio financiero.

En lo referente a la solvencia, aunque no existe un alto riesgo de quiebra en el sector (valores próximos a 1,5, que sería el límite inferior del intervalo ideal), sí habría que mantener 
una cierta vigilancia sobre los parámetros de deuda. La única excepción son las empresas del clúster 5 (sociedades mercantiles distribuidoras), cuya situación de solvencia sí cabría calificar como óptima. Se ha de destacar que los valores correspondientes a la ratio de solvencia para el conjunto de la industria de la alimentación en España para el periodo considerado (1,8 en los tres ejercicios) son más positivos (Banco de España, 2019), quedando patente la situación desfavorable del sector del aceite de oliva.

La escasa solvencia se aprecia principalmente en el clúster de los grupos empresariales exportadores, cuya situación patrimonial es complicada y que, en caso seguir deteriorándose, podría derivar en procesos de quiebra. En un intento por comparar estos datos con los correspondientes a grandes empresas agroalimentarias de otros países, se destaca el trabajo de Katchova y Enlow (2013), que reportan valores de la ratio de solvencia para grandes empresas agroalimentarias norteamericanas para el periodo 2008-2011 cercanos a 2. Este hecho puede apuntar a la posible dificultad a la que se pueden enfrentar los grupos empresariales exportadores españoles para seguir accediendo en condiciones óptimas a los mercados internacionales, tanto para vender sus productos como para conseguir atraer capital para financiar sus inversiones.

Asimismo, también se aprecia una baja solvencia en el clúster 4 (almazaras cooperativas orientadas al mercado nacional), dado que presentó en los ejercicios 2015 y 2016 valores inferiores a 1,5. Sin embargo, se destaca que, en 2017, se logró aumentar el valor de esta ratio a 1,8, lo que, unido a la ratio de endeudamiento de este grupo de empresas en ese ejercicio, da buena cuenta de la adecuada gestión del endeudamiento en el último ejercicio de las empresas de este conglomerado.

Los resultados de la ratio de calidad de la deuda indican que, tanto en el caso del sector en su conjunto, como en cada uno de los conglomerados, la mayor parte de la deuda es a corto plazo, hecho muy evidente en el caso del clúster 5 (sociedades mercantiles distribuidoras), en el que aproximadamente el 90 por ciento corresponde a deuda a corto plazo. Sin embargo, es necesario puntualizar que, en el caso del sector oleícola en su conjunto, el valor de calidad de la deuda en $2017(0,7)$ se corresponde con el valor de esta ratio en el conjunto de la industria de la alimentación (Banco de España, 2019). Los datos de calidad de la deuda deben considerarse, en todo caso, con cautela y no asumir que un elevado porcentaje de deuda a corto plazo es necesariamente un dato negativo; efectivamente, se ha demostrado que para el conjunto de la industria agroalimentaria, las empresas con más obligaciones de pago a corto plazo tienen una mayor probabilidad de presentar elevados niveles de eficiencia (Alarcón, 2008), ya que suelen esforzarse más para poder atender los pagos a corto plazo, lo que repercute positivamente sobre la distribución de recursos.

Por su parte, el coste de la deuda en el sector en su conjunto puede considerarse aceptable (3,6 por ciento en 2017), dado que se sitúa por debajo del actual tipo de interés de mercado de la deuda corporativa, que se encuentra en torno al 5 por ciento, aunque el valor de esta ratio resulta superior al coste de la deuda de la industria de la alimentación en España (2,1 por ciento) (Banco de España, 2019). Diferenciando entre los distintos clústeres, se destacan las empresas correspondientes al clúster 1 (grupos empresariales exportadores) en las que, a pesar de que están consiguiendo una financiación a coste razonable (cercano al tipo de interés de mercado de la deuda), se considera que la deuda asumida es excesiva, lo que hace que hayan ido perdiendo autonomía financiera a lo largo del periodo de tiempo analizado. En un sentido opuesto, resulta igualmente interesante comentar los resultados de las empresas del clúster 4 (almazaras cooperativas orientadas al mercado nacional), donde se advierte una fuerte disminución del coste de la deuda (de un 4,2 por ciento en 2015 a un 1,9 por ciento en 2017), así como el consecuente aumento de su autonomía financiera. 


\section{c) Análisis de las rotaciones}

Tomando como referencia los datos de Banco de España (2019) de 2017 para la industria de la alimentación en lo referente a los valores de rotación del activo no corriente $(2,4)$, activo corriente $(2,4)$, activo total $(1,2)$, existencias $(7,4)$ y clientes $(5,9)$, se puede afirmar que el sector del aceite de oliva utiliza de manera más eficiente los elementos de activo para generar ingresos por ventas, aunque con la excepción de la partida de existencias. Esto es, el sector del aceite de oliva obtiene mayores ingresos por ventas por la utilización de los activos que el conjunto de la industria de la alimentación, siendo este hecho especialmente evidente en el caso de las empresas que conforman el clúster 5 (sociedades mercantiles distribuidoras). Además, por lo general, los datos de las rotaciones en el caso del sector del aceite de oliva han experimentado una evolución positiva a lo largo del período analizado. A pesar de este escenario positivo del sector en su conjunto, es necesario hacer referencia a las empresas del clúster 4 (almazaras cooperativas orientadas al mercado nacional), dado que presentan valores inferiores de las diferentes ratios de rotaciones que el conjunto de la industria de la alimentación y que el sector del aceite de oliva considerado globalmente.

\section{d) Análisis de la cuenta de resultados}

En relación con la evolución de las ventas durante el periodo de estudio, queda patente un aumento de la cifra de ventas en el conjunto del sector (a excepción del clúster 4, almazaras cooperativas orientadas al mercado nacional) y con valores de incremento muy positivos especialmente en las empresas del clúster 2 (Pymes exportadoras) y del clúster 3 (Pequeñas almazaras mercantiles orientadas al mercado nacional). Si se comparan los datos de incremento de ventas del sector (superiores al 8 por ciento en el periodo considerado) con los de la industria de la alimentación, que se sitúan en el 2,1 por ciento en 2016 y 4,6 por ciento en 2017 (Banco de España, 2019), se evidencia el mejor comportamiento del sector del aceite de oliva. Asimismo, en el trabajo de López-Pérez et al. (2018) se reportaron valores de crecimiento de las ventas durante el periodo 2010-2014 que no superaban el 1 por ciento, lo que viene a indicar la mejora del desempeño del sector en cuanto a importe neto de la cifra de ventas se refiere a lo largo del periodo aquí analizado.

El aumento de las ventas en el periodo 2015-2017 ha conllevado un incremento proporcionalmente mayor de los costes variables, aunque la mayor carga de estos costes se ve compensada en el sector con la disminución relativa de los costes fijos, haciendo que el BAII aumente en un 26,8 por ciento en 2017 con respecto al año anterior. Ante este positivo panorama, se ha de destacar que el principal problema del sector es la elevada carga correspondiente a los gastos financieros, consecuencia de la elevada carga de deuda que arrastran las empresas, que lastra de manera importante la evolución de los beneficios antes y después de impuestos. Como se viene comentando, aunque el coste de la deuda del sector es adecuado, sin embargo, el endeudamiento es muy elevado, lo que hace que el total de gastos financieros sea una partida importante de la cuenta de resultados. En cualquier caso, cabe señalar que, a lo largo del periodo analizado, se han logrado reducir estos gastos financieros.

En el caso concreto de las empresas del clúster 1 (grupos empresariales exportadores) y del clúster 3 (pequeñas almazaras mercantiles orientadas al mercado nacional), en 2017 se produce una disminución de los distintos beneficios que componen la cuenta de resultados (BAII, BAI y BN), a pesar de constatarse un incremento de ventas. Este hecho viene motivado por los elevados costes, tanto variables como fijos, a los que tienen que hacer frente estas empresas, lo que hace que incurran en pérdidas.

El principal aspecto a destacar de las almazaras cooperativas orientadas al mercado nacional (clúster 4) es la fuerte caída de las ventas que se produce en 2017, aunque los datos 
de crecimiento del BAII y del BN parecen indicar una disminución más que proporcional de los costes fijos, lo cual resulta positivo.

Por último, se ha de subrayar la buena gestión de los distintos costes y gastos de las sociedades mercantiles distribuidoras (clúster 5), lo que conlleva el aumento de los diferentes beneficios de la cuenta de resultados a lo largo del periodo analizado. Además, un ligero aumento de ventas provoca que el beneficio se dispare, tal y como se refleja en el valor del apalancamiento operativo.

\section{e) Análisis de la rentabilidad}

La rentabilidad del activo o rentabilidad económica del conjunto del sector en el periodo de estudio (valores comprendidos entre 1,6 por ciento y 1,9 por ciento) resulta insuficiente si se compara con el coste de la deuda (de 3,6 por ciento a 5,2 por ciento) y, más aún, con la rentabilidad económica del conjunto de la industria de la alimentación en España en estos mismos años (entre 6,3 por ciento y 6,9 por ciento) (Banco de España, 2019). En este sentido, se destaca que, en el trabajo de López-Pérez et al. (2018), se reportaron datos acerca de la rentabilidad del sector del aceite de oliva comprendidos entre 1,6 por ciento en 2010 y 2,4 por ciento en 2014. De aquí se puede deducir que la rentabilidad económica del sector del aceite de oliva, no solo es baja (inferior a la industria de la alimentación), sino que los bajos valores de esta ratio se han mantenido estables durante los últimos años.

Un análisis más detallado de la rentabilidad económica diferenciando a las empresas según los clústeres de pertenencia, precisa destacar las tasas de rentabilidad económica que se registran en el clúster 5 (sociedades mercantiles distribuidoras), muy superiores a las correspondientes a la industria de la alimentación en su conjunto. En el otro extremo se sitúa el clúster 1 (grupos empresariales exportadores), que presenta valores de la ratio negativos. Es llamativo este último dato: las empresas de mayor tamaño de la muestra presentan los valores más bajos (negativos) de rentabilidad económica. Estos resultados contrastan con anteriores evidencias de la literatura que, por lo general, han encontrado que la dimensión de la empresa es una variable que influye positivamente sobre su rentabilidad económica; por ejemplo, en Zouaghi et al. (2017) para el caso de la industria agroalimentaria en España, o en López-Pérez et al. (2018) para el sector del aceite de oliva. Por su parte, el clúster 2 (pymes exportadoras) y el 3 (pequeñas almazaras mercantiles orientadas al mercado nacional) presentan una evolución positiva de la rentabilidad económica, mostrando valores superiores a sus costes medios de financiación y a la rentabilidad económica correspondiente al sector en su conjunto.

Para analizar la rentabilidad financiera del sector, es necesario calcular la rentabilidad que se espera del mismo, tomando como referencia el Modelo CAPM (Capital Asset Pricing Model) de valoración de activos financieros. Este modelo permite calcular la rentabilidad esperada de cualquier activo financiero $\left(r_{x}\right.$, por ejemplo la de una participación en la propiedad de una empresa del sector analizado) mediante la siguiente expresión (Fama y French, 2004): $r_{x}=$ $r_{f}+\beta_{x}\left(r_{m}-r_{f}\right)$, donde $r_{f}$ expresa la rentabilidad de un activo sin riesgo; $r_{m}$ indica la rentabilidad esperada de mercado; $\left(r_{m}-r_{f}\right)$ determina el valor de la prima de riesgo de mercado; y $\beta_{x}$ expresa la relación entre la rentabilidad de la empresa $x$ y la del conjunto del mercado. No obstante, cuando se trata de activos que presentan dificultades en la transmisión de su propiedad (p. ej., no cotizan en Bolsa), el modelo requiere incluir una prima de liquidez $(P L)$, de tal manera que la expresión anterior quedaría así (Liu, 2006): $r_{x}=\left[r_{f}+\beta_{x}\left(r_{m}-\right.\right.$ $\left.\left.r_{f}\right)\right] \times(1+P L)$, donde $P L$ suele situarse entre 0 por ciento, si la liquidez del activo es muy alta, y 60 por ciento si la liquidez es muy baja. 
En este sentido, debido a que en la muestra de empresas hay tanto empresas grandes y cooperativas que gozan de mayor facilidad para la transmisión de la propiedad (mayor liquidez), como pequeñas y medianas empresas con menor facilidad para la transmisión de la propiedad (menor liquidez), se deben calcular dos rentabilidades esperadas para los propietarios de las empresas que contemplen estas dos realidades.

Por un lado, teniendo en cuenta que actualmente los activos financieros sin riesgo están remunerados incluso por debajo del 1 por ciento, la prima de riesgo de mercado en España está en torno al 6 por ciento y que la beta del sector puede estimarse en el 0,7, la rentabilidad que se esperaría de las empresas grandes (clúster 1) y de las cooperativas (clúster 4) resultaría estar en torno al 5 por ciento. Observando los datos de la tabla 6, se deduce claramente que la rentabilidad financiera de ambos grupos de empresas se sitúa por debajo de la rentabilidad esperada, siendo tremendamente preocupantes los valores negativos del clúster 1 .

Por otro lado, si se incluyera una prima de liquidez en el modelo, que podría ser del 50 por ciento, resultaría que la rentabilidad esperada de las empresas de los clústeres 2 , 3 y 5 (medianas y pequeñas empresas) sería alrededor de un 8 por ciento. Comparando este dato con los valores calculados, se observa que las empresas del clúster 5 (sociedades mercantiles distribuidoras), presentan rentabilidades muy superiores a ese 8 por ciento, mientras que las del clúster 2 (Pymes exportadoras) y 3 (Almazaras cooperativas orientadas al mercado nacional) se sitúan algo por debajo de este valor de referencia.

Por tanto, aunque la rentabilidad financiera del sector en su conjunto (3 por ciento en 2017) se encuentra muy por debajo de su rentabilidad esperada y, a priori, se podría indicar que el sector del aceite de oliva no es atractivo para los inversores, sí conviene señalar que las sociedades mercantiles distribuidoras son una excepción y que están generando un beneficio neto muy elevado en relación con la partida de fondos propios.

En cuanto al apalancamiento financiero, observando la cifra de 2017, se podría afirmar que, teóricamente, si el sector contrajera más deuda, esto podría mejorar la rentabilidad financiera del mismo. Sin embargo, esto no llega a ser del todo recomendable a la vista de los resultados obtenidos correspondientes al análisis del endeudamiento, dado que el sector se encuentra fuertemente endeudado. Sin embargo, también aquí es necesario comentar la situación favorable de las empresas del clúster 5 (sociedades mercantiles distribuidoras): los datos conjuntos de apalancamiento financiero y endeudamiento sugieren que un aumento del nivel de deuda a largo plazo podría resultar útil para conseguir una mayor rentabilidad.

\section{f) Análisis de las necesidades operativas de fondos (NOF)}

La ratio de cobertura de las NOF indica que las necesidades operativas de fondos son superiores al fondo de maniobra durante los tres ejercicios del periodo de análisis, tanto en el caso del sector en su conjunto como en cada uno de los clústeres, a excepción del clúster 5 (sociedades mercantiles distribuidoras). Este hecho vuelve a incidir sobre la falta de liquidez anteriormente comentada. 


\section{TABLA 6. VALORES DE LAS RATIOS ECONÓMICO-FINANCIERAS DEL SECTOR Y DE LOS CLÚSTERES}

\begin{tabular}{|c|c|c|c|c|c|c|c|c|c|c|c|c|c|c|c|c|c|c|c|}
\hline \multirow{2}{*}{ Ratios } & \multirow{2}{*}{$\begin{array}{l}\text { Valores } \\
\text { ideales }\end{array}$} & \multicolumn{3}{|c|}{ SECTOR } & \multicolumn{3}{|c|}{ CLÚSTER 1} & \multicolumn{3}{|c|}{ CLÚSTER 2} & \multicolumn{3}{|c|}{ CLÚSTER 3} & \multicolumn{3}{|c|}{ CLÚSTER 4} & \multicolumn{3}{|c|}{ CLÚSTER 5} \\
\hline & & 2015 & 2016 & 2017 & 2015 & 2016 & 2017 & 2015 & 2016 & 2017 & 2015 & 2016 & 2017 & 2015 & 2016 & 2017 & 2015 & 2016 & 2017 \\
\hline \multicolumn{20}{|l|}{ Liquidez } \\
\hline Liquidez & $1,5-2$ & 1,3 & 1,3 & 1,2 & 1,3 & 1,4 & 1,2 & 1,2 & 1,2 & 1,2 & 1,1 & 1,2 & 1,2 & 1,0 & 1,0 & 1,3 & 2,6 & 1,9 & 1,9 \\
\hline Tesorería & $\sim 1$ & 0,7 & 0,8 & 0,7 & 0,8 & 0,9 & 0,7 & 0,6 & 0,7 & 0,7 & 0,6 & 0,6 & 0,6 & 0,8 & 0,8 & 1,1 & 2,0 & 1,2 & 1,2 \\
\hline Prueba ácida & $0,2-0,3$ & 0,1 & 0,2 & 0,1 & 0,1 & 0,2 & 0,1 & 0,1 & 0,1 & 0,1 & 0,2 & 0,2 & 0,2 & 0,2 & 0,3 & 0,3 & 0,6 & 0,3 & 0,5 \\
\hline F. Maniobra (mill. €) & Positivo & 559 & 571 & 524 & 219 & 276 & 188 & 205 & 161 & 228 & 25 & 30 & 35 & $-4,0$ & $-2,0$ & 12,0 & 104 & 55 & 55 \\
\hline \multicolumn{20}{|l|}{ Endeudamiento } \\
\hline Endeudamiento & $0,4-0,6$ & 0,7 & 0,7 & 0,7 & 0,7 & 0,8 & 0,8 & 0,6 & 0,7 & 0,7 & 0,6 & 0,6 & 0,6 & 0,8 & 0,8 & 0,6 & 0,4 & 0,5 & 0,5 \\
\hline Auton. financiera & $0,7-1,5$ & 0,5 & 0,4 & 0,4 & 0,5 & 0,3 & 0,3 & 0,6 & 0,5 & 0,5 & 0,5 & 0,6 & 0,6 & 0,3 & 0,3 & 0,7 & 1,8 & 1,1 & 1,0 \\
\hline Solvencia & $1,5-2,5$ & 1,5 & 1,4 & 1,4 & 1,5 & 1,3 & 1,3 & 1,6 & 1,5 & 1,5 & 1,6 & 1,6 & 1,6 & 1,3 & 1,3 & 1,8 & 2,8 & 2,1 & 2,0 \\
\hline Calidad de la deuda & Reducido & 0,6 & 0,6 & 0,7 & 0,5 & 0,5 & 0,6 & 0,7 & 0,8 & 0,8 & 0,7 & 0,7 & 0,7 & 0,8 & 0,8 & 0,7 & 1,0 & 1,0 & 0,9 \\
\hline Coste de deuda (\%) & $\leq i_{\text {mercado }}$ & 5,2 & 4,4 & 3,6 & 7,0 & 6,5 & 5,3 & 3,5 & 2,7 & 2,0 & 2,8 & 2,8 & 2,2 & 4,2 & 2,6 & 1,9 & 3,0 & 3,1 & 1,4 \\
\hline Coste medio fin. (\%) & Reducido & 1,6 & 1,5 & 1,3 & 2,4 & 2,4 & 2,0 & 1,1 & 0,9 & 0,8 & 0,9 & 0,8 & 0,6 & 0,8 & 0,5 & 0,6 & 0,1 & 0,1 & 0,1 \\
\hline \multicolumn{20}{|l|}{ Rotaciones } \\
\hline Rotación del ANC & Elevado & 3,1 & 3,5 & 3,8 & 2,7 & 3,4 & 3,5 & 3,6 & 3,8 & 4,4 & 2,1 & 2,4 & 2,6 & 1,9 & 2,2 & 2,1 & 19,3 & 22,5 & 23,2 \\
\hline Rotación del AC & Elevado & 2,7 & 2,8 & 2,9 & 3,4 & 3,4 & 3,2 & 2,6 & 2,7 & 2,7 & 2,0 & 2,4 & 2,3 & 1,1 & 1,3 & 2,2 & 2,3 & 3,3 & 3,3 \\
\hline Rotación Activo total & Elevado & 1,4 & 1,6 & 1,6 & 1,5 & 1,7 & 1,7 & 1,5 & 1,6 & 1,7 & 1,0 & 1,2 & 1,2 & 0,7 & 0,8 & 1,1 & 2,1 & 2,9 & 2,9 \\
\hline Rotación Existencias & Elevado & 6,7 & 7,4 & 6,6 & 9,1 & 10,1 & 7,7 & 5,3 & 6,1 & 5,9 & 4,2 & 4,7 & 4,7 & 5,9 & 5,9 & 12,6 & 9,8 & 8,8 & 8,6 \\
\hline Rotación de Clientes & Elevado & 8,1 & 8,2 & 8,1 & 8,9 & 10,3 & 9,5 & 7,8 & 6,8 & 7,1 & 9,2 & 10,0 & 12,9 & 6,7 & 6,5 & 5,2 & 10,4 & 11,2 & 10,1 \\
\hline \multicolumn{20}{|l|}{ Cuenta Resultados } \\
\hline Crecim. ventas (\%) & Elevado & & 8,2 & 8,5 & & 4,9 & 7,3 & & 8,8 & 18,3 & & 19,4 & 13,6 & & 44,2 & $-62,9$ & & 1,3 & $-0,3$ \\
\hline Evolución MB (\%) & Elevado & & $-3,9$ & $-3,9$ & & 5,3 & $-14,1$ & & $-8,9$ & 4,6 & & 2,8 & 1,9 & & $-34,5$ & $-34,5$ & & $-3,7$ & 15,3 \\
\hline Evolución BAII (\%) & Elevado & & $-13,8$ & 26,8 & & 61,8 & $-29,3$ & & $-26,9$ & 37,4 & & 40,1 & $-12,5$ & & $-581,4$ & $-61,3$ & & 8,5 & 6,7 \\
\hline Evolución BAI (\%) & Elevado & & $-246,9$ & $-193,9$ & & 82,0 & $-65,5$ & & $-18,7$ & 32,8 & & 81,1 & $-14,1$ & & $-334,7$ & $-62,8$ & & $-2,9$ & 1,0 \\
\hline Evolución BN (\%) & Elevado & & $-833,5$ & $-144,8$ & & 246,5 & $-82,2$ & & $-16,6$ & 32,2 & & 116,1 & $-15,2$ & & $-300,9$ & 36,1 & & 0,5 & 0,5 \\
\hline Pto. Equil. (mill. €) & Elevado & 6.486 & 7.708 & 8.903 & 3.215 & 3.619 & 4.464 & 2.440 & 2.941 & 3.676 & 369 & 469 & 551 & 209 & 406 & 115 & 280 & 305 & 268 \\
\hline Cobertura UR & $>1$ & 1,1 & 1,0 & 1,0 & 1,1 & 1,0 & 0,9 & 1,1 & 1,0 & 0,9 & 1,2 & 1,2 & 1,1 & 1,1 & 0,9 & 1,1 & 1,4 & 1,3 & 1,5 \\
\hline Apalanc. operativo & Elevado & & $-1,7$ & 3,1 & & 12,5 & $-4,0$ & & $-3,1$ & 2,0 & & 2,0 & $-0,9$ & & $-13,2$ & 1,0 & & 5,8 & 14,1 \\
\hline \multicolumn{20}{|l|}{ Rentabilidad } \\
\hline Rentab. activo (\%) & Elevado & 1,8 & 1,6 & 1,9 & $-0,5$ & $-0,9$ & $-0,6$ & 4,0 & 2,8 & 3,5 & 3,8 & 5,2 & 4,1 & $-0,3$ & 1,2 & 1,6 & 7,5 & 11,2 & 12,0 \\
\hline Rentab. ventas (\%) & Elevado & 0,2 & $-1,3$ & 0,5 & $-1,4$ & $-4,7$ & $-0,8$ & 1,6 & 1,2 & 1,4 & 1,6 & 2,9 & 2,1 & $-0,7$ & 1,0 & 1,0 & 3,2 & 3,2 & 3,2 \\
\hline Rentab. fra (\%) & Elevado & 0,8 & $-6,8$ & 3,0 & $-7,0$ & $-32,8$ & $-6,1$ & 6,8 & 5,7 & 6,8 & 4,7 & 9,5 & 7,1 & $-2,6$ & 3,9 & 2,8 & 10,4 & 17,9 & 19,0 \\
\hline Margen ventas (\%) & Elevado & 0,0 & 0,0 & 0,0 & 0,0 & 0,0 & 0,0 & 0,0 & 0,0 & 0,0 & 0,0 & 0,0 & 0,0 & 0,0 & 0,0 & 0,0 & 0,0 & 0,0 & 0,0 \\
\hline Apalanc financiero & Elevado & 1,2 & $-2,3$ & 1,7 & 14,2 & 31,3 & 17,7 & 2,2 & 2,6 & 2,5 & 1,9 & 2,4 & 2,3 & 8,3 & 3,7 & 2,0 & 1,9 & 2,1 & 2,1 \\
\hline Autofinan. (mill. €) & Equilibrado & 84 & 4 & 149 & -33 & -130 & 9 & 75 & 77 & 91 & 21 & 30 & 28 & 4 & 11 & 5 & 14 & 14 & 14 \\
\hline Autofin. ventas (\%) & Equilibrado & 1,2 & 0,1 & 1,7 & $-1,0$ & $-3,6$ & 0,2 & 2,8 & 2,6 & 2,6 & 4,5 & 5,5 & 4,5 & 2,0 & 3,3 & 3,8 & 3,6 & 3,6 & 3,6 \\
\hline \multicolumn{20}{|l|}{ NOF } \\
\hline NOF (mill. €) & $<\mathrm{FM}$ & 1.111 & 1.350 & 1.476 & 390 & 506 & 579 & 529 & 594 & 736 & 69 & 75 & 65 & 49 & 82 & 25 & 64 & 41 & 60 \\
\hline Cobertura NOF & $\geq 1$ & 0,5 & 0,4 & 0,4 & 0,6 & 0,6 & 0,3 & 0,4 & 0,3 & 0,3 & 0,4 & 0,4 & 0,5 & $-0,1$ & $-0,0$ & 0,5 & 1,6 & 1,3 & 0,9 \\
\hline
\end{tabular}

Fuente: Elaboración propia. Los valores ideales de las ratios son los propuestos por Amat (2008). 


\section{CONCLUSIONES}

Los resultados de este trabajo han permitido evidenciar la heterogeneidad de la estructura empresarial del sector del aceite de oliva y de su desempeño económico-financiero. Así, mediante el análisis clúster, se han identificado cinco grandes modelos o patrones empresariales en el sector: 1) grupos empresariales exportadores; 2) pymes exportadoras; 3) pequeñas almazaras mercantiles orientadas al mercado nacional; 4) almazaras cooperativas orientadas al mercado nacional; y 5) sociedades mercantiles distribuidoras.

El modelo empresarial más exitoso dentro del sector lo constituye el quinto conglomerado de empresas (sociedades mercantiles distribuidoras). Uno de los principales puntos fuertes de las empresas de este grupo es que gozan en su cuenta de resultados de una estructura de costes bajos, tanto variables como fijos, lo que se puede relacionar con la actividad que predomina en este tipo de sociedades (la distribución del producto). Precisamente este eslabón de la cadena de valor se caracteriza por tener unos bajos costes en comparación con los demás eslabones (producción e industrialización). Asimismo, el incremento de valor añadido propio de la actividad de distribución se traduce en las elevadas cifras de rentabilidad, superiores a la media del sector e, incluso a la correspondiente a la industria de la alimentación en su conjunto.

El primer conglomerado de empresas (grupos empresariales exportadores) constituye la antítesis del caso anterior, evidenciándose como el modelo menos exitoso, con una baja rentabilidad y altas probabilidades de sufrir problemas de solvencia en el futuro. Aunque se trata de grandes grupos empresariales, parece que no logran conseguir las sinergias necesarias para poder hacer frente a los puntos débiles con los que cuentan (p. ej., elevada carga de costes). Esto, junto al hecho de que estas empresas suelen enfocarse a la realización de compraventa del producto a granel y a la venta de aceite de oliva con una calidad media, hace que no se cree el valor añadido necesario para rentabilizar su actividad.

De los restantes conglomerados, el cuarto clúster (almazaras cooperativas orientadas al mercado nacional) es el que sufre de una situación menos favorable, motivada principalmente por su estrategia de comercialización basada en su orientación al mercado nacional, en el que es más que evidente el desequilibrio de la cadena de valor en favor de los grandes operadores comerciales, cuyo poder de negociación es muy elevado.

\section{BIBLIOGRAFÍA}

Alarcón, S. (2008): “Debt financing and efficiency in agricultural firms”, Spanish Journal of Finance and Accounting, vol. 37, $\mathrm{n}^{\circ}$ 138, pp. 211-230. doi: https://dx.doi.org/10.1080/02102412.2008.10779643

Amat, O. (2008): Análisis de estados financieros: fundamentos y aplicaciones, Ediciones Gestión 2000, Barcelona.

Banco de España (2019). "Central de Balances". Recuperado de https://www.bde.es/bde/es/areas/cenbal/

Briz, J., De Felipe, I. y Briz, T. (2010): "Funcionamiento y transparencia en la cadena de valor: aplicación al caso del aceite de oliva en España”, Revista de Estudios Empresariales. Segunda Época, $\mathrm{n}^{\circ}$ 1, pp. 32-53.

Domenech, J., Martinez-Gomez, V. y Mas-Verdú, F. (2014): "Location and adoption of ICT innovations in the agri-food industry”, Applied Economics Letters, vol. 21, n ${ }^{\circ}$ 6, pp. 421424. doi: https://dx.doi.org/10.1080/13504851.2013.864032

Esteban de la Rosa, G. (2015): Internacionalización del sector oleícola a través de la tecnología de la información. Guía para la empresa exportadora de aceite de oliva, Comares, Granada. 
Esteve-Pérez, S., Sanchis-Llopis, A. y Sanchis-Llopis, J. A. (2010): “A competing risks analysis of firms' exit”, Empirical Economics, vol. 38, n 2, pp. 281-304. doi: https://dx.doi.org/10.1007/s00181-009-0266-x

EXTENDA (Agencia Andaluza de Promoción Exterior) (2017): Estudio del sector del aceite de oliva de Andalucía, Consejería de Economía y Conocimiento. Junta de Andalucía, Sevilla.

Fama, E. F. y French, K. R. (2004): “The capital asset pricing model: Theory and evidence”, Journal of Economic Perspectives, vol. 18, $\mathrm{n}^{\circ}$ 3, pp. 25-46. doi: https://dx.doi.org/10.1257/0895330042162430

Galetto, M., Franceschini, F. y Mastrogiacomo, L. (2017): “ISO 9001 certification and corporate performance of Italian companies", International Journal of Quality and Reliability Management, vol. 34, $\mathrm{n}^{\mathrm{0}}$ 2, pp. 231-250. doi: https://dx.doi.org/10.1108/IJQRM-04-2015-0064

Gallardo-Cobos, R. y Sánchez-Zamora, P. (2017): "Olivar y desarrollo rural: las oportunidades derivadas de la diversificación concéntrica", en J. A. Gómez-Limón y M. Parras (Eds.), Economía y comercialización de los aceites de oliva. Factores y perspectivas para el liderazgo español del mercado global (pp. 161-178), Cajamar Caja Rural, Almería.

García-Brenes, D. (2005): "La rentabilidad económica de la industria agroalimentaria en el mercado del aceite de oliva. El caso de Andalucía”, Agroalimentaria, vol. 11, n 21, pp. 43-55.

Gómez-Limón, J. A. y Parras, M. (2017): Economía y comercialización de los aceites de oliva. Factores y perspectivas para el liderazgo español del mercado global, Cajamar Caja Rural, Almería.

Hair, J. F., Black, W. C., Babin, B. J. y Anderson, R. E. (2014): Multivariate Data Analysis, Pearson, Harlow.

Katchova, A. L. y Enlow, S. J. (2013): "Financial performance of publicly-traded agribusinesses”, Agricultural Finance Review, vol. 73, $\mathrm{n}^{\mathrm{o}}$ 1, pp. 58-73. doi: https://dx.doi.org/10.1108/00021461311321311

Kettenring, J. R. (2006): “The practice of cluster analysis”, Journal of Classification, vol. 23, $\mathrm{n}^{\mathrm{o}}$ 1, pp. 3-30. doi: https://dx.doi.org/10.1007/s00357-006-0002-6

Langreo, A. (2010): "La estrategia empresarial en el sector del aceite de oliva y su evolución desde la transición política”, Revista de Estudios Empresariales. Segunda época., vol. $1, \mathrm{n}^{\mathrm{o}}$, pp. 7-31.

Liu, W. (2006): “A liquidity-augmented capital asset pricing model”, Journal of Financial Economics, vol. 82, $\mathrm{n}^{\mathrm{o}}$ 3, pp. 631-671. doi: https://dx.doi.org/10.1016/j.jfineco.2005.10.001

López-Pérez, A., Fernández-López, S., Rodeiro, D. y Li, F. (2018): “Análisis en la relación entre el fondo de maniobra y la rentabilidad: caso del sector oleícola español”, Revista Nacional de Administración, vol. 9, $\mathrm{n}^{0}$ 1, pp. 7-25. doi: https://dx.doi.org/10.22458/rna.v9i1.2102

MAGRAMA (Ministerio de Agricultura, Alimentación y Medio Ambiente) (2012): Estudio de la cadena de valor y formación de precios del aceite de oliva, MAGRAMA, Madrid.

MAPA (Ministerio de Agricultura, Pesca y Alimentación) (2019): Anuario de estadística 2018, MAPA, Madrid.

Martínez-Victoria, M., Maté-Sánchez-Val, M. y Lansink, A. O. (2019): “Spatial dynamic analysis of productivity growth of agri-food companies”, Agricultural Economics, vol. 50, $\mathrm{n}^{\circ}$ 3, pp. 315-327. doi: https://dx.doi.org/10.1111/agec.12486

Maté-Sánchez-Val, M. y Harris, R. (2018): “The paradox of geographical proximity for innovators: A regional study of the Spanish agri-food sector”, Land Use Policy, vol. 73, pp. 458-467. doi: https://dx.doi.org/10.1016/j.landusepol.2018.02.024 
Montegut, Y., Sabaté, P. y Clop, M. (2002): “Análisis económico-financiero de las cooperativas agrarias productoras de aceite de oliva de la DO Garrigues (Lleida, España)", Investigación Agraria, vol. 17, nº 3, pp. 423-440.

Parras, M. y Gómez-Limón, J. A. (2017): "Los retos del sector de los aceites de oliva en España. Estrategias para el liderazgo global", en Economía y comercialización de los aceites de oliva. Factores y perspectivas para el liderazgo español (pp. 15-33), Cajamar Caja Rural, Almería.

Parras, M. y Torres, F. J. (1996): “Una perspectiva regional de la estructura del sector productor de aceite de oliva virgen y su repercusión”, Revista de Estudios Regionales, n 46, pp. 103-135.

Rodríguez-Cohard, J. C. y Parras, M. (2011): “The olive growing agri-industrial district of Jaén and the international olive oils cluster”, The Open Geography Journal, vol. 4, n 1, pp. 55-72. doi: https://dx.doi.org/10.2174/1874923201104010055

Ruiz, C., García, E. y Hernández, M. J. (2013): “Cómo responden a la crisis económica actual las sociedades cooperativas agrarias. El caso de las almazaras cooperativas andaluzas”, REVESCO. Revista de Estudios Cooperativos, $\mathrm{n}^{0}$ 113, pp. 120-149. doi: https://dx.doi.org/10.5209/rev_REVE.2014.v113.43384

Vilar, J. y Cárdenas, J. R. (2016): El sector internacional de elaboración de aceite de oliva: un estudio descriptivo de los 56 países productores, GEA-Centro Internacional de Excelencia para Aceite de Oliva, Úbeda (Jaén).

Zouaghi, F., Sánchez-García, M. y Hirsch, S. (2017): "What drives firm profitability? A multilevel approach to the Spanish agri-food sector”, Spanish Journal of Agricultural Research, vol. 15, n 3, pp. e0117. doi: https://dx.doi.org/10.5424/sjar/2017153-10713 